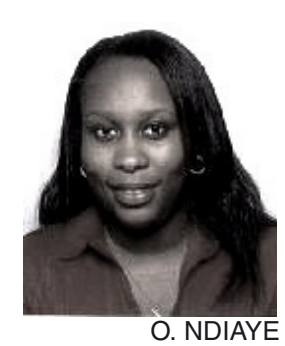

\title{
Caractérisation électromagnétique large bande de couche mince en PZT aux fréquences micro-ondes
}

\section{A broadband electromagnetic characterization of PZT thin film up to microwave frequencies}

\author{
Oumy NDIAYE ${ }^{1,2}$, Michaël CHARLES ${ }^{1}$, Djamel ALLAL $^{1}$ et Bertrand BOCQUET ${ }^{2}$ \\ 1 Laboratoire national de métrologie et d'essais (LNE), 29 avenue Roger Hennequin, 78197 Trappes Cedex, France, Djamel.Allal@lne.fr. \\ 2 Université de Lille 1, Sciences et Technologies, Cité Scientifique, 59655 Villeneuve d'Ascq Cedex, France.
}

\begin{abstract}
Résumé
Une méthode permettant de déterminer les propriétés électromagnétiques d'une couche mince de titano-zirconate de plomb (PZT) est présentée dans cet article. Dans un premier temps nous exposons une méthode large bande permettant l'extraction des propriétés intrinsèques d'un substrat supportant une ligne coplanaire (CPW), puis nous procédons à un étalonnage TRL afin d'extraire la permittivité de la couche mince de PZT intégrée à une topologie coplanaire.
\end{abstract}

MOTS CLÉS : PARAMÈTRES S, LIGNE COPLANAIRE, PZT, PERMITTIVITÉ, PERTES TANGENTIELLES, FERROÉLECTRIQUE.

\begin{abstract}
A method for determining the electromagnetic properties of Lead Zirconate Titanate (PZT) thin films is presented in this paper. At first we introduce a broadband characterization method based on the extraction of the intrinsic properties of substrate supporting a coplanar waveguide and then we perform an accurate on-wafer TRL calibration technique to measure the permittivity of the PZT thin film embedded in a coplanar waveguide.
\end{abstract}

KEY WORDS: S PARAMETERS, COPLANAR WAVEGUIDE, PZT, PERMITTIVITY, LOSS TANGENT, FERROELECTRIC.

\section{Introduction}

La caractérisation des matériaux a été largement étudiée pour des applications numériques au sein des composants électroniques tels que les résonateurs diélectriques, les déphaseurs, les capacités, etc. Les matériaux ferroélectriques constituent une classe importante des matériaux diélectriques utilisés en électronique. Par conséquent, la connaissance de leurs propriétés électromagnétiques est très importante d'une part parce qu'elles constituent une partie de l'ensemble des propriétés spécifiques du matériau et, d'autre part, parce que ces propriétés doivent être maîtrisées dans la perspective d'applications potentielles. Cette étape de caractérisation apparaît cruciale avant l'utilisation de ces matériaux dans des applications micro-ondes car le dimensionnement des circuits est directement lié à la connaissance de leurs propriétés. Le titano-zirconate de plomb (PZT) est un matériau ferroélectrique qui est utilisé pour des applications de la microélectronique telles que les mémoires non volatiles, les détecteurs pyroélectriques, les capteurs mais aussi pour des applications électromécaniques. Il est considéré comme étant un bon candidat pour la fabrication de circuits micro-ondes grâce à sa faible consommation en puissance, son agilité importante, sa facilité d'intégration, notamment en couche mince, et sa permittivité diélectrique qui peut prendre des valeurs exceptionnellement élevées, de 200 à plus de 1000 [1], par l'application d'un champ électrique externe. Ce caractère rend le matériau ferroélectrique particulièrement attractif pour des applications industrielles.

La particularité des matériaux ferroélectriques est leur forte permittivité (de l'ordre de $10^{2}$ à $10^{4}$ ) et leurs pertes diélectriques qui peuvent aussi être élevées (avec tg $\delta$ allant de 0,01 à 1). Or, des pertes trop élevées 
impliquent une dissipation importante de l'énergie électromagnétique. La caractérisation même des matériaux ferroélectriques s'en trouve affectée et requiert une nouvelle approche afin de pouvoir mesurer leurs propriétés diélectriques.

Il existe différentes techniques permettant l'extraction de ces propriétés, certaines sont mono-fréquentielles tandis que d'autres offrent une caractérisation sur une large bande de fréquences [2]. Celle que nous avons retenue est une méthode en ligne de transmission utilisant une ligne coplanaire (CPW) car cette dernière permet une extraction relativement simple de la constante diélectrique mais elle permet aussi une caractérisation large bande. En effet la ligne coplanaire est considérée comme ayant la meilleure topologie pour les couches minces ferroélectriques car elle assure une orientation tangentielle du champ électrique par rapport au plan de la couche mince rendant les conditions de mesure optimales pour cette méthode de caractérisation [3]. En première étape, nous extrayons les propriétés électromagnétiques d'une CPW sur substrat d'alumine afin de valider notre modèle mathématique jusqu'à $110 \mathrm{GHz}$, puis nous extrayons la permittivité du matériau PZT intégré en couche mince dans une ligne coplanaire.

\section{Méthode analytique et processus de caractérisation}

\subsection{La ligne coplanaire et son modèle analytique}

La ligne coplanaire est très utilisée dans les circuits intégrés micro-ondes monolithiques (MMIC), et avec la miniaturisation des circuits et la montée en fréquence des dispositifs, son utilisation s'avère pertinente. Dans le cadre de techniques de caractérisation, notamment pour les méthodes en lignes de transmission, la ligne coplanaire suscite un réel intérêt car elle représente ellemême une cellule de mesure simple, intégrant directement l'échantillon.

Ainsi des relations analytiques permettent de calculer la constante de propagation et l'impédance caractéristique impliquant la détermination des propriétés électromagnétiques.

Les avantages de la ligne coplanaire sont la possibilité de caractérisation sur une large bande de fréquences de façon non destructive et une topologie adaptée aux structures en couches minces. De plus, la ligne coplanaire est un guide d'ondes facile à réaliser qui induit de faibles coûts technologiques et fonctionne dans un mode quasiTEM, peu dispersif malgré la non-homogénéité du milieu (air + substrat) c'est-à-dire que les champs électrique et magnétique sont perpendiculaires à l'axe de la ligne selon lequel s'effectue la propagation.

La figure 1 illustre une ligne coplanaire ainsi que son circuit électrique équivalent pour un élément de ligne, de longueur infinitésimale.

Il est possible d'optimiser les dimensions de la ligne (épaisseur du substrat > largeur du ruban central +2 fois

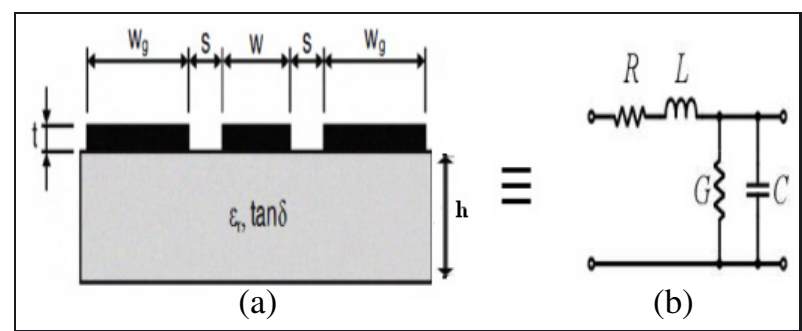

Fig. 1. - Ligne coplanaire (a) et son circuit électrique équivalent (b).

la fente séparant le ruban central et le plan de masse) afin de propager le mode dominant (quasi-TEM) [4], et d'obtenir une meilleure exactitude des paramètres $S$ mesurés.

Un algorithme fondé en partie sur des équations développées par Heinrich [4], a été utilisé afin de calculer les paramètres $R, L, G$ et $C$ de l'équivalent circuit de la ligne coplanaire. Ces paramètres permettent d'obtenir par le calcul d'autres paramètres caractérisant la ligne coplanaire tels que l'impédance caractéristique $Z_{c}$, la constante de propagation $\gamma$ et les paramètres $S$. Les expressions de l'impédance caractéristique et de la constante de propagation sont décrites par les équations (1) et (2) ci-dessous :

$$
\begin{aligned}
Z_{c} & =\sqrt{\frac{R+j L \omega}{G+j C \omega}} \\
\gamma & =\sqrt{(R+j L \omega)(G+j C \omega)}
\end{aligned}
$$

où $\omega$ est la pulsation en $\mathrm{rd} \cdot \mathrm{s}^{-1}$.

L'obtention de l'impédance caractéristique et de la constante de propagation permet d'obtenir la matrice ABCD (3) suivante :

$$
\mathbf{A B C D}=\left(\begin{array}{cc}
\cosh (\gamma L) & Z_{c} \sinh (\gamma L) \\
\frac{\sinh (\gamma L)}{Z_{c}} & \cosh (\gamma L)
\end{array}\right)
$$

Puis les paramètres $S$ (matrice $\mathbf{S}$ ) sont obtenus à partir de la matrice ABCD. La figure 2 présente les paramètres $S_{11}$ et $S_{21}$ obtenus analytiquement pour une ligne coplanaire sur substrat d'alumine.

En considérant un mode de propagation quasi-TEM, la permittivité relative peut être déduite selon l'expression (4) suivante [3] :

$$
\varepsilon_{r}=\frac{\frac{C}{2 \varepsilon_{0}}-\text { Fup }}{\text { Flow }},
$$

où $C$ est la composante capacitive de la ligne coplanaire, $\varepsilon_{0}$ la permittivité diélectrique du vide, et Fup et Flow sont des paramètres dépendant des dimensions géométriques de la ligne coplanaire, plus amplement détaillés dans la référence [4].

La figure 3 représente la permittivité relative calculée pour une ligne coplanaire sur substrat d'alumine. 


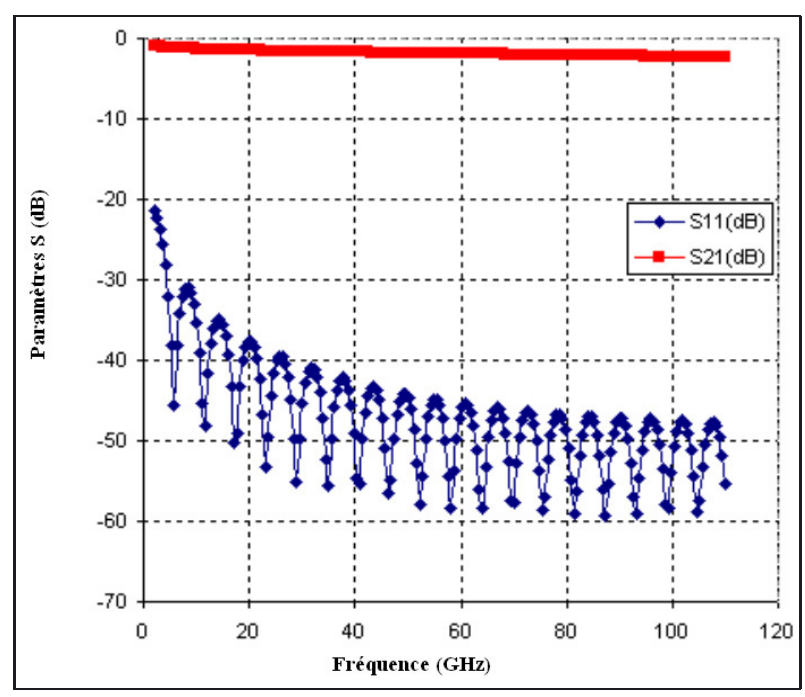

Fig. 2. - Modules, exprimés en décidels (dB), des paramètres $S$ en réflexion et en transmission d'une ligne coplanaire sur substrat d'alumine ( $W=60 \mu \mathrm{m}, S=28 \mu \mathrm{m}, L=11047 \mu \mathrm{m}$, $t=500 \mathrm{~nm}, h=635 \mu \mathrm{m})$.

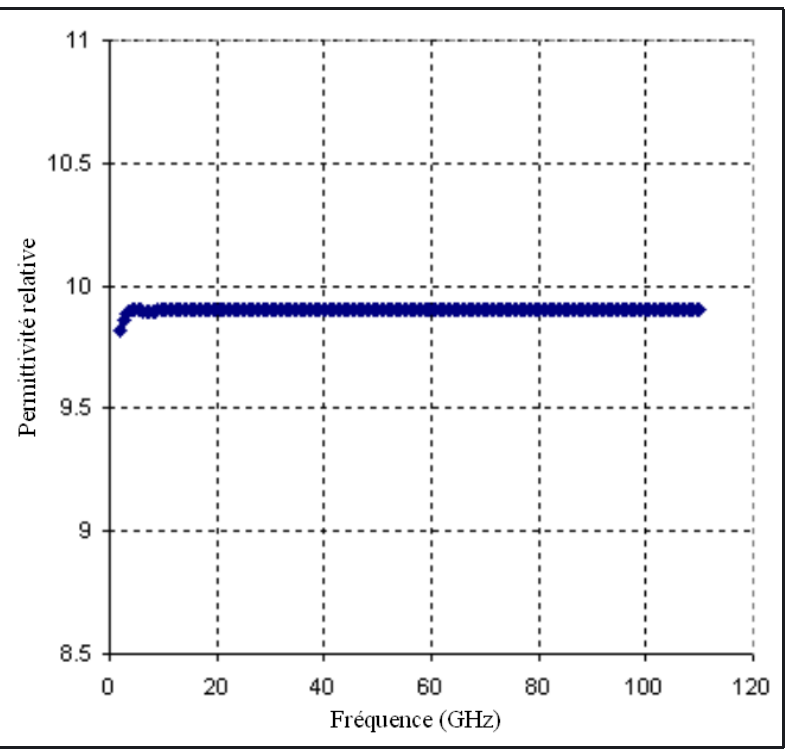

Fig. 3. - Permittivité relative calculée pour une ligne coplanaire sur substrat d'alumine $(W=60 \mu \mathrm{m}, S=28 \mu \mathrm{m}$, $L=11047 \mu \mathrm{m}, t=500 \mathrm{~nm}, h=635 \mu \mathrm{m})$.

On constate que la valeur obtenue est constante sur toute la bande de fréquence et qu'elle correspond à la permittivité relative donnée par le fabricant $\left(\varepsilon_{\mathrm{r}}=9,9\right)$. On observe une décroissance de la permittivité relative pour les fréquences basses, due à l'effet de peau qui entraine une désadaptation de la ligne coplanaire à ces fréquences.

\subsection{Caractérisation des lignes coplanaires sur substrat d'alumine à l'analyseur de réseau vectoriel (VNA)}

En amont de la fabrication, il est nécessaire de modéliser la ligne coplanaire étudiée afin de définir ses dimensions géométriques permettant une adaptation à $50 \Omega$ et un fonctionnement optimal sur la bande de fréquences considérée. Ainsi nous avons modélisé, sur le substrat d'alumine, un jeu de cinq lignes avec des longueurs transposées au modèle de Doerner et al. [5].

Une première étape consiste à calculer les paramètres résistif $R$ et inductif $L$ de l'équivalent circuit de la ligne coplanaire à partir des dimensions de cette dernière selon le modèle établit par Heinrich [3].

L'étape suivante a pour but de déterminer la constante de propagation de notre topologie coplanaire sur substrat d'alumine. Pour ce faire, nous mesurons les paramètres $S$ bruts (analyseur de réseau vectoriel non étalonné) d'un jeu de cinq lignes coplanaires présentant les mêmes dimensions hormis la longueur.

À partir de ces paramètres $S$ bruts et des facteurs de réflexion des ports 1 et 2 de l'analyseur de réseau vectoriel, nous déterminons une nouvelle matrice $\mathbf{M}$, dite matrice de mesure [6] :

$$
\left.\begin{array}{l}
\mathbf{M}= \\
M_{11}=\frac{S_{21 m} S_{12 m}-S_{11 m} S_{22 m}}{S_{21 m}\left(1-S_{22 m} \Gamma_{F}\right)} M_{12}=\frac{S_{11 m}-S_{12 m} S_{21 m} \Gamma_{F}}{S_{21 m}\left(1-S_{22 m} \Gamma_{F}\right)} \\
M_{21}=\frac{-S_{22 m}+S_{12 m} S_{21 m} \Gamma_{R}}{S_{21 m}\left(1-S_{22 m} \Gamma_{F}\right)} \quad M_{22}=\frac{1-S_{12 m} S_{21 m} \Gamma_{F} \Gamma_{R}}{S_{21 m}\left(1-S_{22 m} \Gamma_{F}\right)}
\end{array}\right)
$$

où $\Gamma_{R}$ et $\Gamma_{F}$ correspondent respectivement aux facteurs de réflexion des ports 1 et 2 de l'analyseur de réseau, et $S_{i j m}$ (avec $i, j=1,2$ ) sont les paramètres $S$ bruts mesurés du dispositif sous test.

En associant deux à deux les matrices $\mathrm{M}$ des cinq lignes coplanaires, nous déterminons, par la méthode TL (Thru-Line), la constante de propagation sur toute la bande de fréquences. Une analyse de la constante de phase et de la constante d'atténuation sur les différents couples de lignes du substrat est effectuée afin de trouver, à chaque point de fréquence le couple de lignes optimal.

Enfin, avec la constante de propagation et les paramètres $R$ et $L$ du circuit équivalent à la ligne coplanaire, nous déterminons le paramètre $C$ qui contient la permittivité du substrat.

Le synoptique de la figure 4 résume les différentes étapes permettant d'aboutir à l'extraction de la permittivité relative du substrat.

Les mesures sont réalisées sous pointes à l'aide d'une station de mesure Süss Microtec PM8 associée à un analyseur de réseau Agilent $8510 \mathrm{XF}$, couvrant les fréquences de $2 \mathrm{GHz}$ à $110 \mathrm{GHz}$. Un exemple de mesure est montré sur la figure 5 pour une ligne de longueur $11,047 \mathrm{~mm}$. On constate des pertes plus importantes en hautes fréquences car les effets de couplage capacitif entre le ruban central et les plans de masse de la ligne coplanaire deviennent très importants.

La figure 6 montre des résultats concordants entre la simulation et la mesure. En effet on constate que la valeur de la permittivité relative mesurée s'avoisine de la valeur 


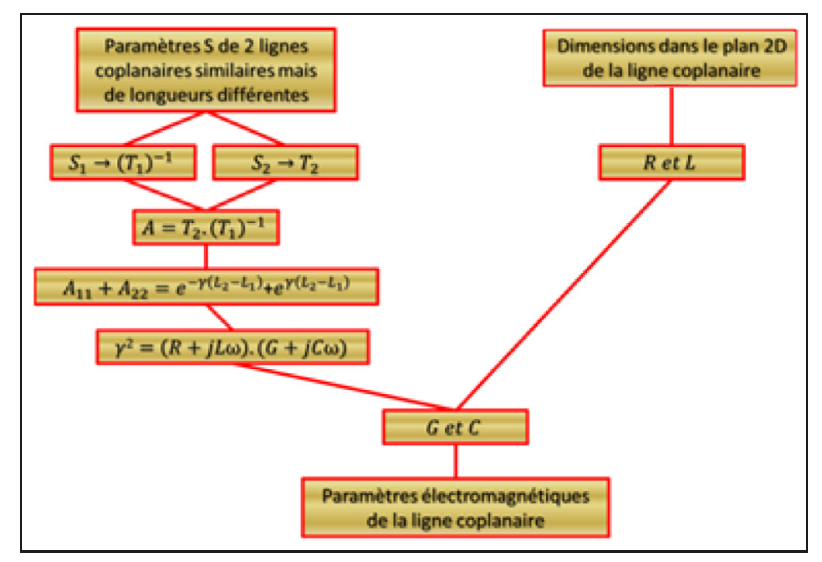

Fig. 4. - Synoptique explicitant les différentes étapes permettant d'aboutir à l'extraction de la permittivité relative du substrat.

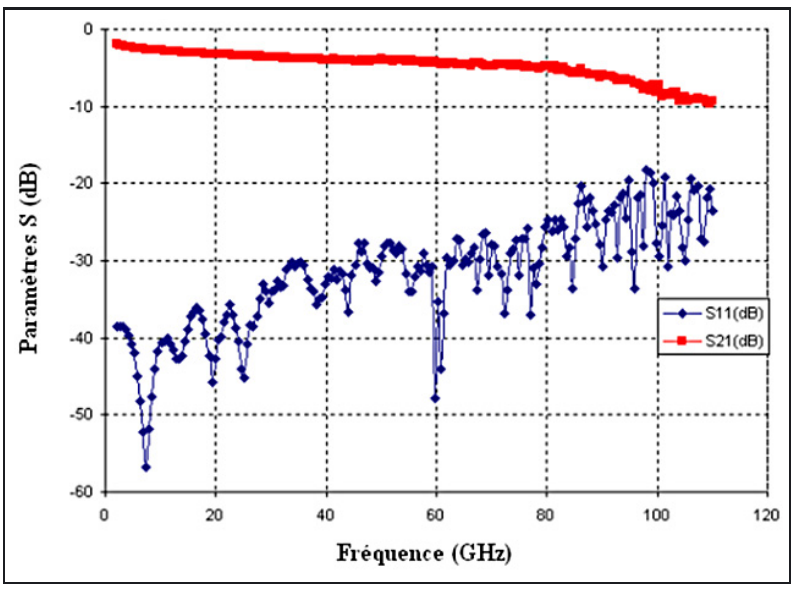

Fig. 5. - Modules, exprimés en décibels (dB), de paramètres $S$ en réflexion et transmission pour une ligne coplanaire sur substrat d'alumine $(W=60 \mu \mathrm{m}, S=28 \mu \mathrm{m}, L=11,047 \mathrm{~mm}$, $t=500 \mathrm{~nm}, h=635 \mu \mathrm{m})$.

trouvée en simulation, de même ces résultats concordent aussi avec la valeur donnée par le fabricant du matériau $\left(\varepsilon_{\mathrm{r}}=9,9\right)$. Les oscillations observables au début et à la fin de la bande de fréquence étudiée s'expliquent par la désadaptation des lignes coplanaires aux basses fréquences due à l'effet de peau et aux effets dispersifs en haute fréquence non pris en compte dans le modèle quasi-statique.

Le calcul d'incertitude se fait généralement suivant la méthode de propagation des variances préconisée par le Guide pour l'expression de l'incertitude de mesure (GUM) [7]. Cette méthode permet de déterminer les incertitudes à partir du processus de mesure, c'est-à-dire de prendre en compte les incertitudes dues aux appareils de mesure et celles du modèle mathématique utilisé pour extraire les résultats. La propagation des incertitudes requiert la dérivation du modèle mathématique appliqué au processus de mesure en fonction des différentes variables.

De manière générale, ce calcul s'appuie sur la loi de propagation des incertitudes sur la base d'un développement de Taylor appliqué aux équations du système.

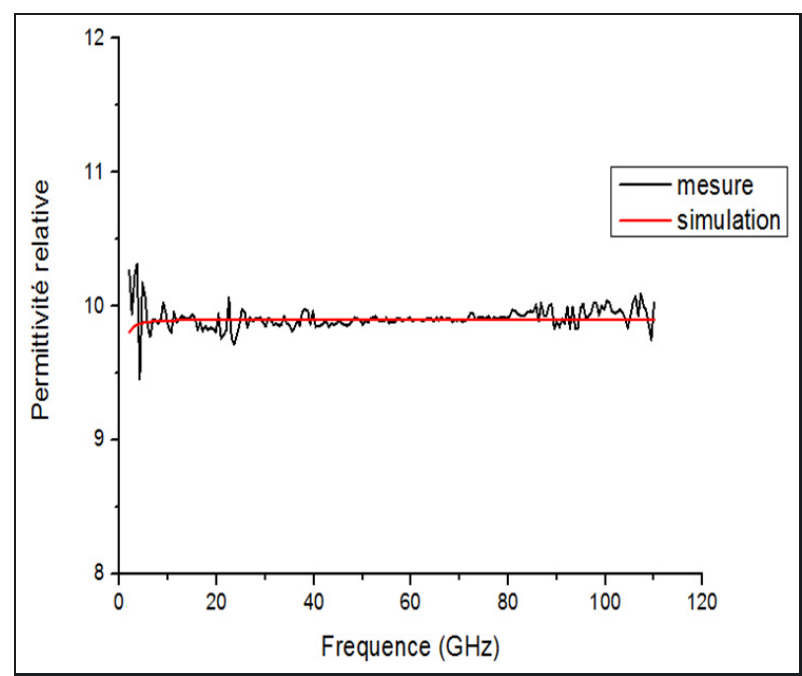

Fig. 6. - Mesure et simulation de la permittivité relative de l'alumine.

Compte tenu de la complexité des systèmes d'équations, la méthode de Monte-Carlo est une alternative intéressante pour l'évaluation de l'incertitude de mesure. En effet avec cette méthode, il ne s'agit plus de propager l'incertitude mais la densité de probabilité des grandeurs d'entrées afin d'obtenir la densité de probabilité associée au mesurande [8].

La méthode de Monte-Carlo permet de reconstituer artificiellement un phénomène aléatoire. En réalité, il s'agit de simuler un échantillon fictif de réalisations de ce phénomène à partir d'hypothèses sur les variables aléatoires.

Afin de mettre en œuvre la méthode de Monte-Carlo à notre modèle, nous appliquons une loi normale à l'ensemble des paramètres dimensionnels de la ligne coplanaire et des paramètres $S$ mesurés dont nous connaissons les moyennes et les incertitudes. Nous utilisons le calculateur MATLAB pour réaliser un grand nombre de tirages sur chacun des paramètres selon une loi normale. Afin d'obtenir une moyenne des tirages sur une loi normale qui soit proche de 0 , nous devons réaliser 300000 tirages.

Nous obtenons au final 300000 résultats de la permittivité relative du substrat dont nous pouvons déduire une moyenne et une incertitude.

La figure 7 présente les incertitudes associées aux résultats expérimentaux de la permittivité relative de l'alumine.

Les résultats expérimentaux en ce qui concerne la permittivité relative de l'alumine présente de faibles incertitudes. La plus grande valeur sur l'incertitude de mesure se situe en basse fréquence (à $2 \mathrm{GHz}$ ), cela est dû à la désadaptation. Mis à part le premier point de fréquence, l'incertitude de mesure est très faible sur le résultat de la permittivité de l'alumine avec une incertitude de mesure qui varie de 0,01 à 0,02 entre $10 \mathrm{GHz}$ et $110 \mathrm{GHz}$.

Les résultats obtenus nous permettent de valider l'algorithme élaboré avec Matlab et de pouvoir conclure, 


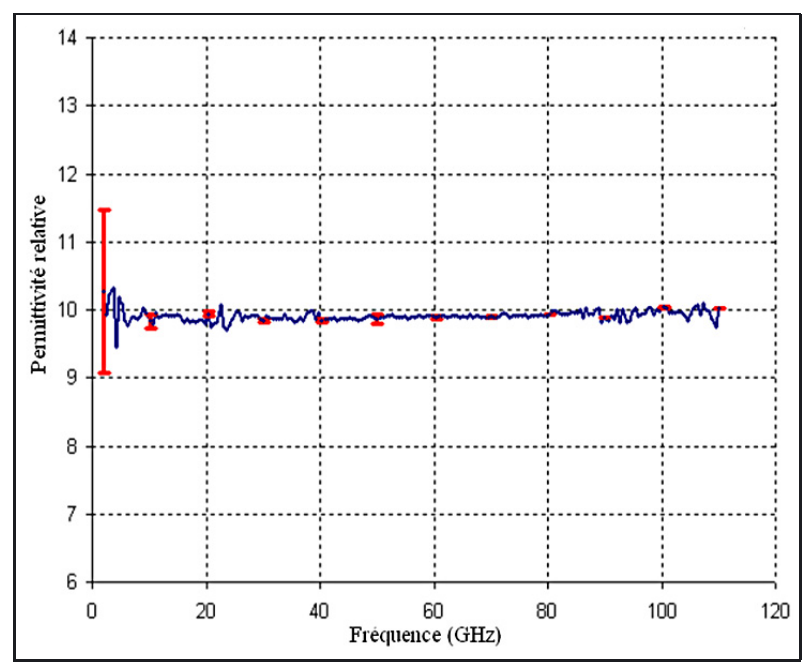

Fig. 7. - Mesure de la permittivité relative de l'alumine et son incertitude associée.

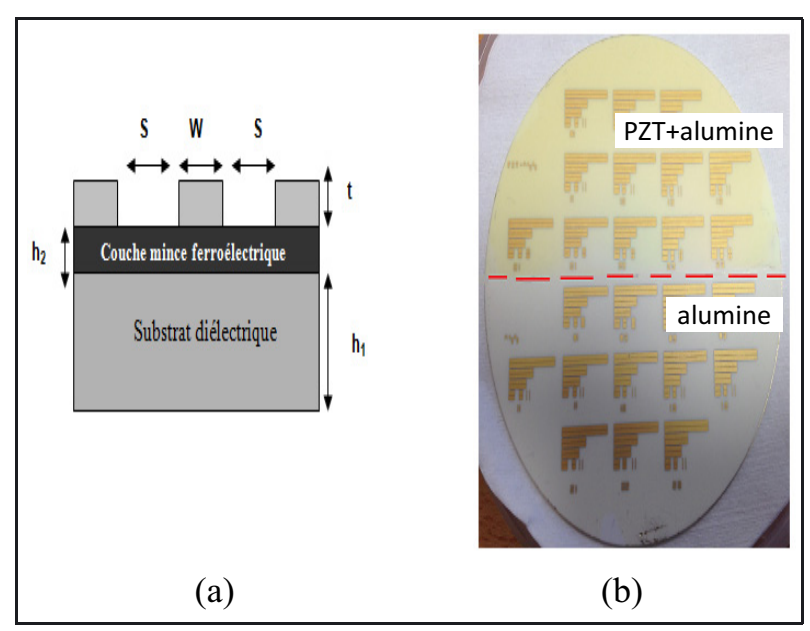

Fig. 8. - Structure coplanaire intégrant une couche mince ferroélectrique (a) et le dispositif de caractérisation (b).

qu'à ce stade, nous sommes en mesure d'extraire la permittivité d'un substrat donné. L'étape suivante consiste à insérer un matériau tel qu'un ferroélectrique entre les plans de la ligne coplanaire et le substrat et d'en extraire les propriétés électromagnétiques.

\section{Extraction des propriétés électromagnétiques de la couche mince ferroélectrique de PZT}

\subsection{Préparation des échantillons}

Les premiers résultats expérimentaux ont été obtenus avec une épaisseur de $400 \mathrm{~nm}$ de couche de PZT déposé par dépôt physique en phase vapeur à $650{ }^{\circ} \mathrm{C}$ sur la moitié d'un substrat d'alumine d'épaisseur $635 \mu \mathrm{m}$, sur laquelle seront déposées par la suite des lignes avec une métallisation en or d'épaisseur $500 \mathrm{~nm}$. Sur la partie du substrat ne contenant pas la couche mince ferroélectrique, des lignes coplanaires, de dimensions géométriques identiques à celles du substrat d'alumine caractérisé précédemment, sont réalisées afin de pouvoir effectuer un étalonnage de l'analyseur de réseau (fig. 8).

\subsection{Extraction des propriétés électromagnétiques du PZT}

Afin d'extraire la permittivité de la couche mince ferroélectrique, nous avons étalonné l'analyseur de réseau vectoriel selon une méthode multiline TRL en utilisant la partie du substrat d'alumine sans couche mince ferroélectrique.

La méthode TRL (Thru-Reflect-Line) est la méthode d'étalonnage la mieux adaptée pour la caractérisation de composants utilisés pour les mesures sous pointes. Cette technique repose sur la mesure de trois éléments :

- Le Thru qui correspond à la connexion directe entre les deux ports de l'analyseur de réseau, sa matrice $\mathbf{S}$ $\left(S_{11}=S_{22}=0 ; S_{12}=S_{21}=1\right)$ est connue si les impédances caractéristiques des deux ports sont identiques.

- Le Reflect est un quadripôle virtuel à fort coefficient de réflexion constitué de deux charges identiques mais inconnues (généralement court-circuit ou circuit ouvert).

- La Line correspond à une ligne de transmission adaptée avec une matrice $\mathbf{S}$ partiellement connue $\left(S_{11}=\right.$ $S_{22}=0$ ) et un facteur de transmission inconnu déterminé lors de l'étalonnage.

L'étalonnage de l'analyseur de réseau se fait avec Multical qui est un logiciel élaboré sur la base d'un algorithme développé par le NIST (National Institute of Standards and Technologies) qui délivre une estimation de l'incertitude (de type statistique) de ses propres résultats. L'algorithme de traitement repose sur le calcul de la moyenne des estimations de Gauss-Markov [9].

Nous pouvons ainsi obtenir les paramètres $S$ corrigés et la constante de propagation, paramètres nécessaires pour déterminer la permittivité relative de la couche mince de ferroélectrique.

La figure 9 présente un facteur de transmission plus faible que celui présenté à la figure 5, à cause de la présence de la couche mince.

L'expression de la constante diélectrique du matériau PZT, dans cette configuration multicouche, est décrite comme suit [10] :

$$
\begin{aligned}
\varepsilon_{2} & =\frac{1}{q_{2}}\left(\varepsilon_{\mathrm{eff}}-1-q_{1}\left(\varepsilon_{1}-1\right)\right)+\varepsilon_{1}=\varepsilon_{2}^{\prime}-j \varepsilon_{2}^{\prime \prime} \\
q_{i} & =\frac{1}{2} \frac{K\left(k_{i}\right)}{K\left(k_{i}^{\prime}\right)} \frac{K\left(k_{0}^{\prime}\right)}{K\left(k_{0}\right)}, \quad i=1,2 \\
\operatorname{tg} \delta_{2} & =\frac{\varepsilon_{2}^{\prime \prime}}{\varepsilon_{2}^{\prime}}
\end{aligned}
$$

où $\varepsilon_{1}$ est la permittivité relative du substrat, $\varepsilon_{\text {eff }}$ est la permittivité effective du système (alumine $+\mathrm{PZT}+$ air) et $q_{1}$ et $q_{2}$ sont les facteurs de remplissage, $K\left(k_{0}\right)$ et $K\left(k_{i}\right)$ sont les intégrales elliptiques du premier ordre, $K\left(k_{0}^{\prime}\right)$ et $K\left(k_{i}^{\prime}\right)$ sont les intégrales elliptiques du second ordre et $k_{0}, k_{i}$ 


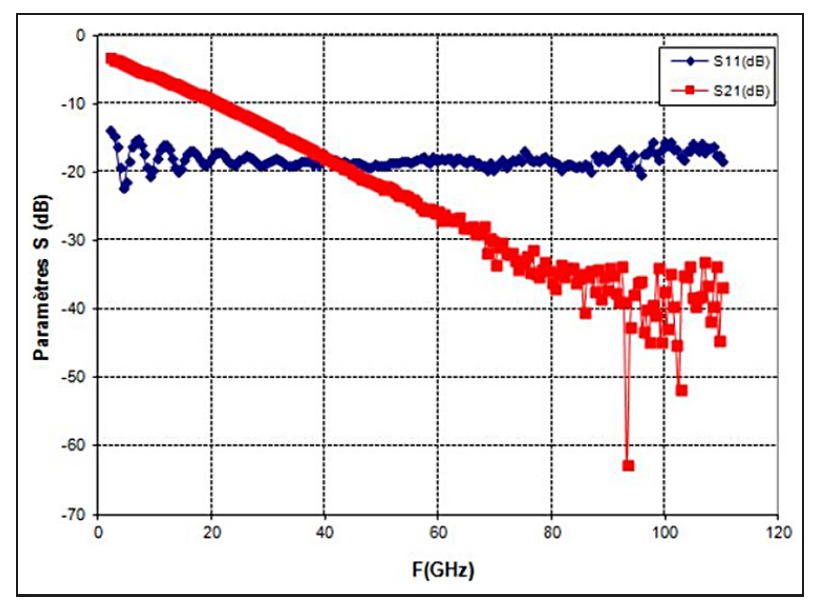

Fig. 9. - Modules, exprimés en décibels (dB), des facteurs de transmission et de réflexion d'une ligne coplanaire intégrant une couche mince de PZT.

sont des coefficients qui dépendent des dimensions géométriques de la ligne coplanaire.

Dans notre cas, les dimensions de la ligne coplanaire (largeur du ruban central $W$ et espace entre le ruban central et le plan de masse $S$ ) sont très grandes devant l'épaisseur de la couche mince $\left(h_{2}\right)$, cela implique que la valeur de $k_{2}$ devient très petite $\left(<10^{-45}\right)$ induisant des erreurs numériques lors du calcul des fonctions elliptiques. Afin de s'affranchir de ces erreurs, nous utilisons une approximation asymptotique pour le rapport des intégrales elliptiques [11] :

Pour $0 \leq k_{i} \leq 0,707$,

$$
\frac{K\left(k_{i}\right)}{K\left(k_{i}^{\prime}\right)}=\frac{\pi}{\ln \left[\frac{2\left(1+\sqrt{\sqrt{1-k_{i}^{2}}}\right)}{1-\sqrt{\sqrt{1-k_{i}^{2}}}}\right]}
$$

Quand $k_{2}$ tend vers 0,

$$
\frac{K\left(k_{i}\right)}{K\left(k_{i}^{\prime}\right)}=\frac{\pi}{\ln \left[\frac{16}{k_{i}^{2}}\right]}
$$

alors,

$$
q_{2}=\frac{1}{2} \frac{\pi}{\ln \left[\frac{16}{k_{2}^{2}}\right]} \frac{K\left(k_{0}^{\prime}\right)}{K\left(k_{0}\right)}
$$

Les résultats obtenus pour la permittivité relative et les pertes tangentielles de la couche mince de PZT d'épaisseur $400 \mathrm{~nm}$ sont présentés dans les figures 10 et 11 .

En ce qui concerne la permittivité relative du matériau PZT d'épaisseur $400 \mathrm{~nm}$, on observe une décroissance « rapide » entre $2 \mathrm{GHz}$ et $20 \mathrm{GHz}$, ce qui démontre

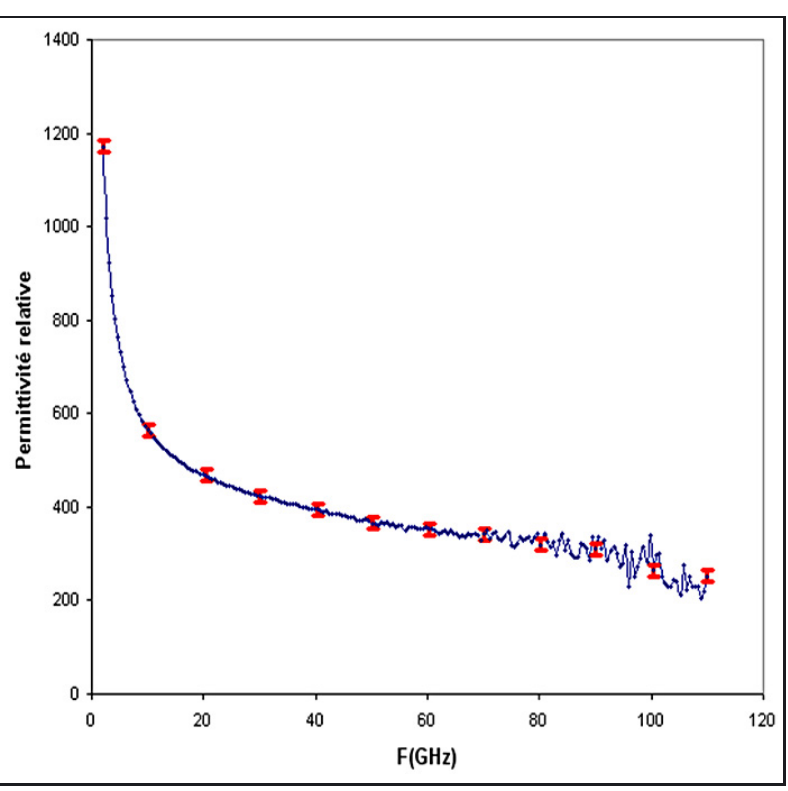

Fig. 10. - Résultats de mesure de la permittivité relative d'une couche mince de PZT (400 nm) et leurs incertitudes associées.

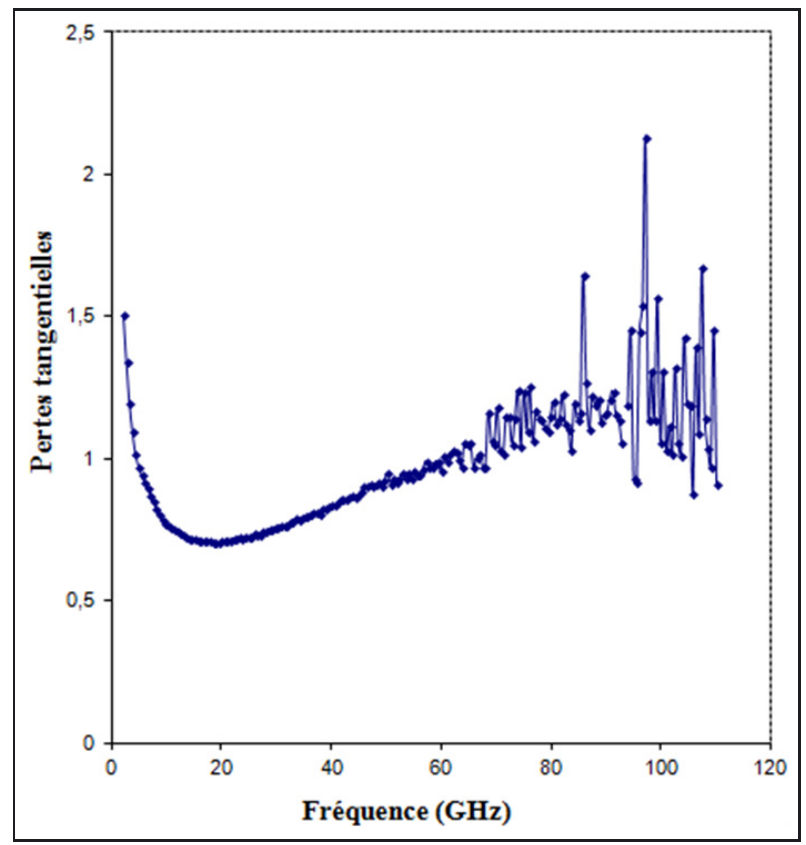

Fig. 11. - Résultats de mesure des pertes tangentielles d'une couche mince de PZT (400 nm).

les limites de conception design de nos lignes coplanaires dans les basses fréquences. Pour que la méthode soit opérante à ces fréquences, il faudrait une épaisseur de métallisation plus importantes pour permettre au courant de surface de circuler pleinement dans la métallisation et ainsi supprimer le phénomène d'effet de peau, et des dimensions de la ligne coplanaire permettant d'être adaptée à $10 \mathrm{GHz}$. Cependant, ces modifications engendreraient des problèmes au-delà de $30 \mathrm{GHz}$ car nous verrions apparaître une désadaptation ainsi que des effets de couplages capacitifs trop importants entre le ruban central et les masses de la ligne coplanaire. 
On constate que la permittivité relative varie de 1171 à 200 entre $2 \mathrm{GHz}$ et $110 \mathrm{GHz}$, ce qui correspond à l'état de l'art [1]. Les incertitudes de mesure de la permittivité relative de la couche mince de PZT sont relativement faibles et constantes sur toute la bande de fréquences, ces dernières restant inférieures à 7 entre $2 \mathrm{GHz}$ et $110 \mathrm{GHz}$.

Comme attendu, la permittivité observée pour la couche mince de PZT est très élevée mais les pertes tangentielles (fig. 11) sont également élevées variant ainsi de 1,5 à 1,3 entre $2 \mathrm{GHz}$ et $110 \mathrm{GHz}$.

L'analyse et l'interprétation des pertes tangentielles présentées sur la figure 11 sont beaucoup plus complexes. En effet l'un des inconvénients de la méthode destructive est qu'il est impossible d'opérer une mesure différentielle permettant de s'affranchir des pertes métalliques, des pertes par rayonnement et des pertes diélectriques. Plusieurs phénomènes peuvent affecter la détermination des pertes tangentielles de la couche mince ferroélectrique :

- les erreurs aléatoires dues au rapport signal sur bruit de l'analyseur de réseau qui ne sont pas complètement supprimées par l'étalonnage TRL, mais réduites ;

- les pertes par rayonnement et les pertes d'ondes de surfaces qui sont effectives aux hautes fréquences (> $100 \mathrm{GHz})$ [12] ;

- les pertes par conduction (métalliques) et les pertes diélectriques du substrat;

- les courants de surface liés à l'épaisseur de métallisation.

\section{Conclusion}

Une technique de caractérisation large bande de couches minces ferroélectriques de matériau PZT allant jusqu'à $110 \mathrm{GHz}$ a été mise en exergue dans cet article. Les résultats obtenus pour la permittivité sont encourageants et nous avons démontré la possibilité d'intégrer un matériau PZT sur des substrats d'alumine pour des applications en microélectronique. Le principal défi reste de diminuer les pertes diélectriques [13, 14].

De nouveaux axes de recherche permettent d'envisager une diminution de ces pertes à l'aide de procédés de fabrication et de dépôt innovants, tels que :

- le dopage métallique ou diélectrique;

- la fabrication d'une hétéro-structure multicouche.

Par conséquent, la prochaine étape de nos travaux se concentrera sur une analyse numérique pour éviter les erreurs d'étalonnage aux basses fréquences et sur l'établissement d'un bilan complet d'incertitude pour finaliser la traçabilité métrologique des matériaux ferroélectriques aux fréquences micro-ondes.

\section{Références}

[1] Haccart T., Cattan E. et Remiens D., "Dielectric, ferroelectric and piezoelctric properties of sputtered PZT thin films onSi substrates: influence of film thickness and orientation", Semicond. Phys., Quantum Eletron. Optoelectron., 5, 1, 2002, 78-88.

[2] BARTLEy P.G. et BEgley S.B., "A new technique for the determination of the complex permittivity and permeability of materials", Instrum. Meas. Technology Conference (I2MTC), IEEE, 3-6 mai 2010, Austin, Etats-Unis, 54-57, DOI: $10.1109 /$ IMTC.2010.5488184.

[3] Queffelec P., Laur V., Chevalier A., Le Floch J., PasserieuX D. Cros D., Mandrangeas V., Le Febvrier A., Deputier S., GuillouX-Viry M., Houzet G., Lacrevaz T., Bemond C. et Fléchet B., "Intercomparison of permittivity measurement techniques for ferroelectric thin layers", J. Appl. Phys., 115, 2014, 024103.

[4] HEINRICH W., "Quasi-TEM description of MMIC coplanar lines including condutor loss effect", IEEE Trans. Microwave Theory Techniques, 41, 1, 1993.

[5] DOERnER R. et RUMiansteV A., "Verification of the wafer-level LRM+Calibration technique for GaAs applications up to $110 \mathrm{GHz}$, 65th ARFTG Conference, Long Beach, juin 2005.

[6] Allal D., «Raccordement des mesures de DAS », Rapport d'étude, LNE, février 2012.

[7] «Évaluation des données de mesure - Guide pour l'expression de l'incertitude de mesure », JCGM 100:2008, www.bipm.org.

[8] Desenfant M., Fischer N. et Blanquart B., «Évaluation de l'incertitude en utilisant les simulations de Monte Carlo », $13^{e}$ Congrès international de métrologie, Paris, France, octobre 2013.

[9] Williams D., Wang C. et Arz U., "An Optimal Multiline TRL Calibration Algorithm", Microwave Symposium Digest, IEEE MTT-S International, 2003, Vol. 3.

[10] Spartak Gevorgian, "Ferroelectrics in Microwave Devices, Circuits and Systems", pp. 247-260.

[11] GuPta K., GARg R., BAHL I. et Bhartia P., "Microstrip lines and slotlines", $2^{\text {nd }}$ edition: Artech House, Boston MA, 1998, chap. 7.

[12] Kassem H., Vigneras V. et Lunet G., "Non-destructive dielectric characterization of thin ferroelectric films materials using coplanar line structure", Integr. Ferroelectr., 94, 2007, 82-93.

[13] Reymond V., Michau D., Payan S. et Maglione M., "Improving the dielectric losses of $(\mathrm{Ba}, \mathrm{Sr}) \mathrm{TiO}_{3}$ thin films using a $\mathrm{SiO}_{2}$ buffer layer", Ceram. Int., 30, 7, 2004, 10851087.

[14] Chong K., Kong L., Cheng L., YAn L., TAN C., Yang T., ONG C. et OsIPOWIK T., "Improvement of dielectric loss tangent of $\mathrm{Al} 2 \mathrm{O} 3$ doped $\mathrm{Ba} 0.5 \mathrm{Sr} 0.5 \mathrm{TiO} 3$ thin films for tunable microwave devices", J. Appl. Phys., 95, 3, 2004.

Article reçu le 8 janvier 2015, version révisée reçue le 13 novembre 2015. 